\title{
Bifurcation Analysis of Periodic Motions Originating from Regular Precessions of a Dynamically Symmetric Satellite
}

\author{
E. A. Sukhov
}

We deal with motions of a dynamically symmetric rigid-body satellite about its center of mass in a central Newtonian gravitational field. In this case the equations of motion possess particular solutions representing the so-called regular precessions: cylindrical, conical and hyperboloidal precession. If a regular precession is stable there exist two types of periodic motions in its neighborhood: short-periodic motions with a period close to $2 \pi / \omega_{2}$ and long-periodic motions with a period close to $2 \pi / \omega_{1}$ where $\omega_{2}$ and $\omega_{1}$ are the frequencies of the linearized system $\left(\omega_{2}>\omega_{1}\right)$.

In this work we obtain analytically and numerically families of short-periodic motions arising from regular precessions of a symmetric satellite in a nonresonant case and long-periodic motions arising from hyperboloidal precession in cases of third- and fourth-order resonances. We investigate the bifurcation problem for these families of periodic motions and present the results in the form of bifurcation diagrams and Poincaré maps.

Keywords: Hamiltonian mechanics, satellite dynamics, bifurcations, periodic motions, orbital stability

\section{Introduction}

The motion of a dynamically symmetric satellite in a circular orbit was studied in many papers $[1-4,6-16]$. One of the important subjects of study in satellite dynamics is periodic motions. Since equations of motion in satellite dynamics usually cannot be integrated analytically, a relevant problem is to develop asymptotical and numerical methods for computing and studying periodic motions. The problem of numerical computation of periodic motions was

Received June 20, 2019

Accepted October 20, 2019

This work was carried out at the Moscow Aviation Institute (National Research University) within the framework of the state assignment (project No. 3.3858.2017/4.6).

Egor A. Sukhov

sukhov.george@gmail.com

Moscow aviation institute (National Research University)

Volokolamskoe sh. 4, GSP-3, A-80, Moscow, 125993 Russia

RUSSIAN JOURNAL OF NONLINEAR DYNAMICS, 2019, 15(4), 593-609 
addressed in [17] where an approach that allows a reduction of the boundary-value problem to a simpler Cauchy problem was proposed. Numerical methods based on this approach were developed in [18-22] where motions arising from the satellite's hyperboloidal precession were computed analytically and numerically and their linear orbital stability was studied.

In this paper we compute analytically and numerically families of short-periodic motions originating from regular precessions of a symmetric satellite in a nonresonant case and families of long-periodic motions originating from hyperboloidal precession in the case of third- and fourth-order resonances. We consider the bifurcation problem of these motions.

\section{Formulation of the problem}

We consider the motion of a satellite around its center of mass $O$ which moves in a circular orbit in a central Newtonian gravitational field at angular velocity $\omega_{0}$. The satellite is considered to be a dynamically symmetric rigid body with principal moments of inertia $J_{1}, J_{2}$ and $J_{3}$ $\left(J_{1}=J_{2}\right)$. To describe the satellite's motion around its center of mass, we introduce an orbital reference frame $O X Y Z$ and a mobile reference frame $O x y z$. Axes $O X, O Y$ and $O Z$ are aligned with transversal and normal vectors to the orbit and with the radius vector of the satellite's center of mass, respectively. Axes $O x, O y$ and $O z$ are aligned with the satellite's principal axes of inertia. The relative position of these reference frames is defined by Euler's angles $\psi, \theta, \varphi$. In this case the system possesses a cyclical coordinate $\varphi$ and its respective impulse $p_{\varphi}$ retains constant value. Following [3, 7], the equations of motion of a dynamically symmetric satellite can be written in a canonical form with the following Hamiltonian:

$$
\begin{aligned}
H & =\frac{p_{\psi}^{2}}{2 \sin ^{2} \theta}+\frac{p_{\theta}^{2}}{2}-\left(\frac{\gamma \cos \theta}{\sin ^{2} \theta}+\cos \psi \cot \theta\right) p_{\psi}- \\
& -\sin \psi p_{\psi}+\frac{1}{2} \gamma^{2} \cot ^{2} \theta+\gamma \frac{\cos \psi}{\sin \theta}+\frac{1}{2} \delta \cos ^{2} \theta,
\end{aligned}
$$

where $p_{\psi}$ and $p_{\theta}$ are dimensionless impulses corresponding to variables $\psi$ and $\theta, \delta=3\left(J_{3} / J_{1}-1\right)$ and $\gamma=\frac{J_{3}}{J_{1}} \frac{r_{0}}{\omega_{0}}$ are dimensionless parameters and $r_{0}$ is the projection of the satellite's absolute angular velocity along its principal axis $O z$. The independent variable is a true anomaly $\nu=\omega_{0} t$. The equations of motions with Hamiltonian (2.1) possess particular solutions

$$
\begin{gathered}
\theta_{0}=\frac{\pi}{2}, \quad \psi_{0}=\pi, \quad p_{\theta_{0}}=0, \quad p_{\psi_{0}}=0, \\
\sin \theta_{0}=\frac{\gamma}{\delta-1}, \quad \psi_{0}=0, \quad p_{\theta_{0}}=0, \quad p_{\psi_{0}}=\delta \sin \theta_{0} \cos \theta_{0} . \\
\theta_{0}=\frac{\pi}{2}, \quad \cos \psi_{0}=-\gamma, \quad p_{\theta_{0}}=\sin \psi_{0}, \quad p_{\psi_{0}}=0,
\end{gathered}
$$

known as regular precessions [2, 3].

Solution (2.2) exists for all values of $\gamma, \delta$ and is known as cylindrical precession. It defines a relative equilibrium of the satellite in the orbital reference frame when its principal axis $O z$ is aligned with the normal vector to the satellite's orbit and describes a cylinder in absolute space (Fig. 1a). Figure 1b presents a stability diagram for cylindrical precession where gray areas represent domains of instability and white areas represent domains of stability.

Solution (2.3) exists for $|\gamma| \leqslant|\delta-1|$ (subdomains $I-I I I$ in Fig. 1d) and is known as conical precession. In this case the satellite's principal axis $O z$ remains orthogonal to the transversal 


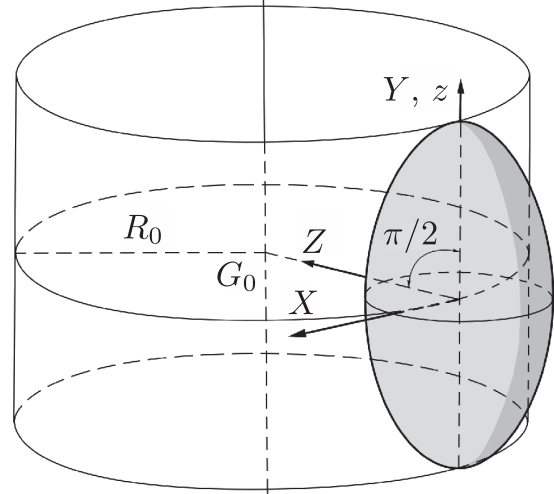

(a)

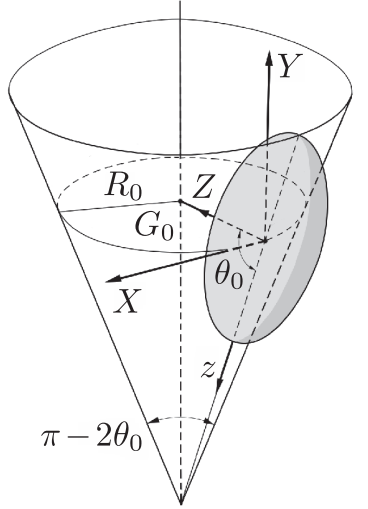

(c)

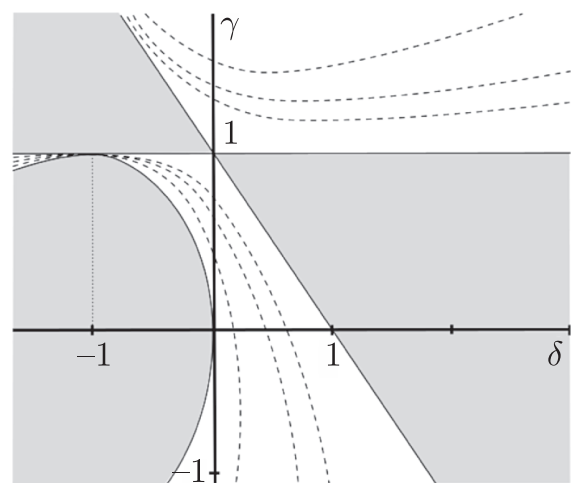

(b)

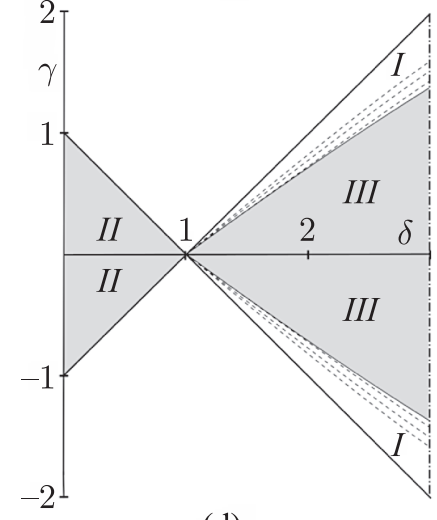

(d)
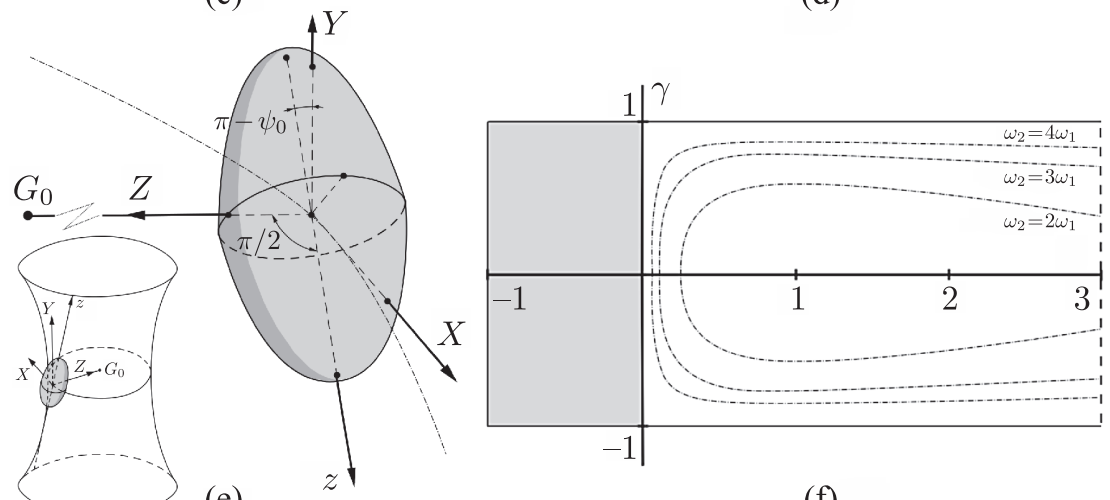

(f)

Fig. 1. Regular precessions of a symmetric satellite (a, c, d) and their existence and stability domains in the plane of problem parameters $\gamma, \delta$ (b, e, f). Here $G_{0}$ is the gravitating center, $Y$ is aligned with the normal vector to the satellite's orbit and $X$ is aligned with the transversal vector.

vector of the orbital reference and describes a cone in absolute space with an angle $\pi-2 \theta_{0}$ at its tip (Fig. 1c). Figure 1d presents a stability diagram for conical precession where gray areas represent domains of instability and white areas represent domains of stability.

Solution (2.4) is known as hyperboloidal precession and defines a relative equilibrium of the satellite in the orbital reference frame when its principal axis $O z$ lies in a plane perpendicular to the radius vector of the satellite's center of mass and remains at a constant angle $\pi-\psi_{0}$ to the normal vector (Fig. 1d). The satellite's principal axis $O z$ describes a hyperboloid in absolute space. Hyperboloidal precession exists for $|\gamma| \leqslant 1$ and is Lyapunov stable if $\delta>0$ [4] (Fig. 1f). 


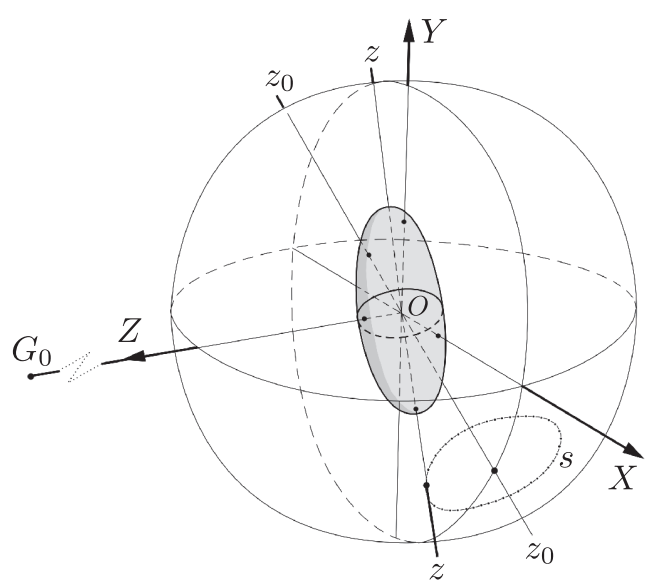

Fig. 2. Periodic motion in the neighborhood of hyperboloidal precession. Here $G_{0}$ is the gravitating center, $z_{0}$ is the position of the satellite's figure axis $O z$ in the case of hyperboloidal precession and $s$ is the trace of the satellite's principle axis $O z$ on the unit sphere.

Two types of periodic motions exist in the neighborhood of a stable regular precession: short-periodic motions with period close to $2 \pi / \omega_{2}$ and long-periodic motions with period close to $2 \pi / \omega_{1}$, respectively. These periodic motions represent oscillations of the satellite's principle axis $O z$ in the neighborhood of regular precession (Fig. 2) and can be obtained as small parameter power series of the oscillation amplitude $c=c(\Delta h)$ where $\Delta h$ is a deviation of the energy constant $h$ from its value for a corresponding regular precession. Here $\omega_{1,2}$ are the frequencies of the linearized system

$$
\omega_{1,2}=\sqrt{F_{1}^{j} \mp \sqrt{F_{2}^{j}}}
$$

where coefficients $F_{1}^{j}$ and $F_{2}^{j}(j=\Gamma, Z, K)$ in $(2.5)$ corresponding to cylindrical $(Z)$, conical $(K)$ and hyperboloidal $(\Gamma)$ precession are given by the following formulae:

$$
\begin{aligned}
F_{1}^{\Gamma} & =\frac{\delta+1}{2}, F_{2}^{\Gamma}=\frac{(\delta-1)^{2}+4 \gamma^{2} \delta}{4}, \\
F_{1}^{Z} & =\frac{1}{2} \gamma^{2}-\gamma+\frac{1}{2} \delta+1, \quad F_{2}^{Z}=\frac{1}{4} \gamma^{4}-\gamma^{3}+\gamma^{2}-2 \gamma \delta+\delta+\frac{1}{2} \gamma^{2} \delta+\frac{1}{4} \delta^{2}, \\
F_{1}^{K} & =\frac{1}{2(\delta-1)^{2}}\left[1+5 \delta^{2}+\delta^{2} \gamma^{2}+3 \delta \gamma^{2}-2 \delta^{3}-4 \delta\right], \\
F_{2}^{K} & =\frac{1}{4(\delta-1)^{4}}\left[1-4 \gamma^{2} \delta^{5}+\left(\gamma^{2}+1\right)^{2} \delta^{4}+\left(-4+10 \gamma^{2}+6 \gamma^{4}\right) \delta^{3}+\right. \\
& \left.+\left(9 \gamma^{4}+6-10 \gamma^{2}\right) \delta^{2}+\left(-4+2 \gamma^{2}\right) \delta\right] .
\end{aligned}
$$

\section{Analytical computation of periodic motions originating from hyperboloidal precession}

In this paragraph, following [14-16, 23], we obtain families of periodic motions originating from regular precessions of a dynamically symmetric satellite in the form of small parameter power series of amplitude $c$. 


\subsection{Nonresonant case}

To obtain the periodic motions originating from regular precessions of a satellite in a nonresonant case, we follow the method developed by Lyapunov [5] and introduce local coordinates

$$
\psi=\psi_{0}+\xi_{1}, \quad \theta=\theta_{0}+\xi_{2}, \quad p_{\psi}=p_{\psi_{0}}+\eta_{1}, \quad p_{\theta}=p_{\theta_{0}}+\eta_{2}
$$

in the neighborhood of a regular precession and expand the Hamiltonian (2.1) into series of $\xi_{i}, \eta_{i}$ $(i=1,2)$

$$
H=H_{2}^{j}+H_{3}^{j}+H_{4}^{j}+\ldots,
$$

where $H_{k}^{j}(j=Z, K, \Gamma)$ are polynomials of degree $k$ in variables $\xi_{i}, \eta_{i}$. We then apply a canonical transformation

$$
\begin{aligned}
& \xi_{1}=\kappa_{1}^{j} A_{11}^{j} x_{1}+\kappa_{2}^{j} A_{12}^{j} x_{2}, \quad \xi_{2}=\kappa_{1}^{j} A_{21}^{j} y_{1}+\kappa_{2}^{j} A_{22}^{j} y_{2}, \\
& \eta_{1}=\kappa_{1}^{j} A_{31}^{j} x_{1}+\kappa_{2}^{j} A_{32}^{j} x_{2}, \quad \eta_{2}=\kappa_{1}^{j} A_{41}^{j} y_{1}+\kappa_{2}^{j} A_{42}^{j} y_{2},
\end{aligned}
$$

which transforms (3.2) to the following normal form:

$$
\begin{gathered}
H=\frac{1}{2} \omega_{1}\left(x_{1}^{2}+y_{1}^{2}\right)+\frac{1}{2} \omega_{2}\left(x_{2}^{2}+y_{2}^{2}\right)+\sum_{n_{1}+n_{2}+m_{1}+m_{2}=3} h_{n_{1} n_{2} m_{1} m_{2}} x_{1}^{n_{1}} x_{2}^{n_{2}} y_{1}^{m_{1}} y_{2}^{m_{2}}+ \\
+\sum_{n_{1}+n_{2}+m_{1}+m_{2}=4} h_{n_{1} n_{2} m_{1} m_{2}} x_{1}^{n_{1}} x_{2}^{n_{2}} y_{1}^{m_{1}} y_{2}^{m_{2}}+\ldots,
\end{gathered}
$$

where $h_{n_{1} n_{2} m_{1} m_{2}}$ are constant coefficients dependent upon $\gamma$ and $\delta$. The coefficients $\kappa_{1}^{j}, \kappa_{2}^{j}$, $A_{i 1}^{j}, A_{i 2}^{j}(i=1, \ldots, 4, k=Z, K, \Gamma)$ of the transformation (3.3) are given by the following formulae:

$$
\begin{aligned}
& A_{11}^{Z}=\frac{1}{\omega_{1}}\left(1-\gamma-\delta+\omega_{1}^{2}\right) \omega_{1}, A_{12}^{Z}=\frac{1}{\omega_{2}}\left(1-\gamma-\delta+\omega_{2}^{2}\right) \\
& A_{21}^{Z}=A_{22}^{Z}=\gamma-2 \\
& A_{31}^{Z}=\left(2 \gamma-1-\gamma^{2}-\delta+\omega_{1}^{2}\right), A_{32}^{Z}=\left(2 \gamma-1-\gamma^{2}-\delta+\omega_{2}^{2}\right) \\
& A_{41}^{Z}=\frac{1}{\omega_{1}}\left(\gamma+\delta-\gamma \omega_{1}^{2}-1+\omega_{1}^{2}\right), A_{42}^{Z}=\frac{1}{\omega_{2}}\left(\gamma+\delta-\gamma \omega_{2}^{2}-1+\omega_{2}^{2}\right), \\
& \kappa_{i}^{Z}=A^{Z}\left(\omega_{i}\right), \quad i=1,2 \\
& A^{Z}(\omega)=\frac{1}{\omega} \gamma^{3}+(\delta-4) \gamma^{2}+\left(6-2 \omega^{2}-2 \delta\right) \gamma-3+ \\
& +2 \delta+\omega^{4}+\delta^{2}+2 \omega^{2}-2 \delta \omega^{2}, \\
& A_{11}^{K}=\frac{\delta-1}{\omega_{1} \omega_{1} \gamma^{2}}\left(\omega_{2} \delta^{2}-2 \omega_{2} \delta+\omega_{2}+\omega_{2} \delta \omega_{1}^{2}-\omega_{2} \gamma^{2}-\omega_{2} \omega_{1}^{2}\right) \\
& A_{12}^{K}=\frac{\delta-1}{\omega_{1} \omega_{1} \gamma^{2}}\left(\omega_{1} \delta^{2}-2 \omega_{1} \delta+\omega_{1} \delta \omega_{2}^{2}-\omega_{1} \gamma^{2}+\omega_{1}-\omega_{1} \omega_{2}^{2}\right) \\
& A_{21}^{K}=A_{22}^{K}=\delta+1 \\
& A_{31}^{K}=\frac{1}{(\delta-1)^{2}}\left(\omega_{1}^{2}-4 \delta^{2}-\delta^{2} \gamma^{2}-3 \delta \gamma^{2}+2 \delta^{3}+2 \delta-2 \omega_{1}^{2} \delta+\omega_{1}^{2} \delta^{2}\right), \\
& A_{32}^{K}=\frac{1}{(\delta-1)^{2}}\left(\omega_{2}^{2}+\omega_{2}^{2} \delta^{2}-2 \omega_{2}^{2} \delta-\delta^{2} \gamma^{2}-3 \delta \gamma^{2}+2 \delta^{3}+2 \delta-4 \delta^{2}\right),
\end{aligned}
$$




$$
\begin{aligned}
& A_{41}^{K}=\frac{1}{\omega_{1} \omega_{1} \gamma^{2}}\left(\omega_{2} \omega_{1}^{2}-\omega_{2}-2 \omega_{2} \delta \omega_{1}^{2}+\omega_{2} \omega_{1}^{2} \delta^{2}-\right. \\
& \left.-\omega_{2} \gamma^{2} \omega_{1}^{2}-3 \omega_{2} \delta^{2}-\omega_{2} \delta \gamma^{2}+\omega_{2} \delta^{3}+3 \omega_{2} \delta+\omega_{2} \gamma^{2}-\omega_{2} \gamma^{2} \omega_{1}^{2} \delta\right) \\
& A_{42}^{K}=\frac{1}{\omega_{1} \omega_{1} \gamma^{2}}\left(\omega_{1} \delta^{3}+3 \omega_{1} \delta+\omega_{1} \gamma^{2}+\omega_{1} \omega_{2}^{2}+\omega_{1} \omega_{2}^{2} \delta^{2}-\right. \\
& \left.-2 \omega_{1} \delta \omega_{2}^{2}-\omega_{1}-\omega_{1} \gamma^{2} \omega_{2}^{2}-\omega_{1} \gamma^{2} \omega_{2}^{2} \delta-3 \omega_{1} \delta^{2}-\omega_{1} \delta \gamma^{2}\right) \\
& \kappa_{i}^{K}=\frac{1}{(\delta-1) \gamma^{2} \omega_{i}} A\left(\omega_{i}\right), \quad i=1,2 \\
& A^{K}(\omega)=2 \omega^{2}-\omega^{4}-\gamma^{2} \delta^{4}-2 \gamma^{2} \delta^{3}+\left(8-2 \omega^{2}\right) \gamma^{2} \delta^{2}+\gamma^{4} \delta^{2}+3 \gamma^{4} \delta+ \\
& +\left(5+3 \omega^{4}-8 \omega^{2}\right) \delta+\left(1-2 \omega^{2}\right) \gamma^{2}+\left(12 \omega^{2}-3 \omega^{4}-10\right) \delta^{2}+\delta^{5}+ \\
& +\left(-6+4 \omega^{2}\right) \gamma^{2} \delta+\left(-5+2 \omega^{2}\right) \delta^{4}+\left(\omega^{4}+10-8 \omega^{2}\right) \delta^{3}-1 . \\
& A_{11}^{\Gamma}=\frac{\delta-\omega_{1}^{2}}{\omega_{1}}, A_{12}^{\Gamma}=\frac{\delta-\omega_{2}^{2}}{\omega_{2}}, \\
& A_{21}^{\Gamma}=A_{22}^{\Gamma}=\gamma, \\
& A_{31}^{\Gamma}=\delta-\omega_{1}^{2}, A_{\Gamma}^{Z}=\delta-\omega_{2}^{2}, \\
& A_{41}^{\Gamma}=-\frac{\gamma \delta}{\omega_{1}}, A_{42}^{Z}=-\frac{\gamma \delta}{\omega_{2}}, \\
& \kappa_{i}^{\Gamma}=\sqrt{\frac{\omega_{i}}{\omega_{i}^{4}+\gamma^{2} \delta-2 \delta \omega_{i}^{2}+\delta^{2}}}, \quad i=1,2 .
\end{aligned}
$$

The expressions $(3.5),(3.6)$ and $(3.7)$ correspond to the cases of cylindrical $(Z)$, conical $(K)$ and hyperboloidal $(\Gamma)$ precession, respectively.

We then apply another canonical transformation $x_{i}, y_{i} \rightarrow q_{i}, p_{i}(i=1,2)$ which transforms (3.4) to the form

$$
\begin{aligned}
K & =\frac{1}{2} \omega_{1}\left(q_{1}^{2}+p_{1}^{2}\right)+\frac{1}{2} \omega_{2}\left(q_{2}^{2}+p_{2}^{2}\right)+ \\
& +a_{20}\left(q_{1}^{2}+p_{1}^{2}\right)^{2}+a_{11}\left(q_{1}^{2}+p_{1}^{2}\right)\left(q_{2}^{2}+p_{2}^{2}\right)+a_{02}\left(q_{2}^{2}+p_{2}^{2}\right)^{2}+K^{(5)},
\end{aligned}
$$

where $a_{20}, a_{11}, a_{02}$ are constant coefficients dependent upon $\gamma$ and $\delta$ and $K_{5}$ are terms of order 5 and higher. The canonical system with Hamiltonian (3.8) admits a family of short-periodic motions

$$
q_{2}=c \sin \Omega_{2}\left(\nu-\nu_{0}\right), \quad p_{2}=c \cos \Omega_{2}\left(\nu-\nu_{0}\right), \quad q_{1}=p_{1}=0,
$$

with period $T_{2}=2 \pi / \Omega_{2}$ where

$$
\Omega_{2}=\omega_{2}+4 c^{2} a_{02}+O\left(c^{4}\right) .
$$

The parameter $c$ in (3.9) represents the small amplitude of oscillations of the satellite's axis $O z$ in the neighborhood of a regular precession. Returning to the initial variables $\psi, \theta, p_{\psi}, p_{\theta}$, we obtain the following asymptotic expressions for the families of short-periodic motions originating from regular precessions:

$$
\begin{aligned}
\psi & =\psi_{0}+c \kappa_{2} A_{12}^{j} \sin \Omega_{2}\left(\nu-\nu_{0}\right)+O\left(c^{2}\right), \\
p_{\psi} & =p_{\psi_{0}}+c \kappa_{2} A_{32}^{j} \cos \Omega_{2}\left(\nu-\nu_{0}\right)+O\left(c^{2}\right), \\
\theta & =\theta_{0}+c \kappa_{2} A_{22}^{j} \cos \Omega_{2}\left(\nu-\nu_{0}\right)+O\left(c^{2}\right), \\
p_{\theta} & =p_{\theta_{0}}+c \kappa_{2} A_{42}^{j} \sin \Omega_{2}\left(\nu-\nu_{0}\right)+O\left(c^{2}\right),
\end{aligned}
$$


where coefficients $\kappa_{1}^{j}, \kappa_{2}^{j}, A_{i 1}^{j}, A_{i 2}^{j}(i=1, \ldots, 4, k=Z, K, \Gamma)$ are given by (3.5)-(3.7). In presenting further results, we will refer to the families (3.10) of short-periodic motions originating from cylindrical, conical and hyperboloidal precessions as $Z_{s}, K_{s}$ and $\Gamma_{s}$, respectively.

The canonical system with Hamiltonian (3.8) also admits a family of long-periodic motions

$$
q_{2}=p_{2}=0, \quad q_{1}=c \sin \Omega_{1}\left(\nu-\nu_{0}\right), \quad p_{1}=c \cos \Omega_{1}\left(\nu-\nu_{0}\right)
$$

with period $T_{1}=2 \pi / \Omega_{1}\left(T_{1}>T_{2}\right)$ where $\Omega_{1}=\omega_{1}+4 c^{2} a_{20}+O\left(c^{4}\right)$. Upon returning to the initial variables $\psi, \theta, p_{\psi}, p_{\theta}$ expressions (3.11) give the following series of amplitude $c$ :

$$
\begin{aligned}
\psi & =\psi_{0}+c \kappa_{1} A_{11}^{j} \sin \Omega_{1}\left(\nu-\nu_{0}\right)+O\left(c^{2}\right), \\
p_{\psi} & =p_{\psi_{0}}+c \kappa_{1} A_{31}^{j} \cos \Omega_{1}\left(\nu-\nu_{0}\right)+O\left(c^{2}\right), \\
\theta & =\theta_{0}+c \kappa_{1} A_{21}^{j} \cos \Omega_{1}\left(\nu-\nu_{0}\right)+O\left(c^{2}\right), \\
p_{\theta} & =p_{\theta_{0}}+c \kappa_{1} A_{41}^{j} \sin \Omega_{1}\left(\nu-\nu_{0}\right)+O\left(c^{2}\right),
\end{aligned}
$$

which represent the long-periodic motions originating from regular precessions in a nonresonant case.

\subsection{Third-order resonance}

To analytically obtain the families of periodic motions originating from hyperboloidal precession in the neighborhood of third-order resonance $\left(\omega_{2}=2 \omega_{1}\right)$, we apply a canonical transformation $\psi, \theta, p_{\psi}, p_{\theta} \rightarrow \xi_{1}, \xi_{2}, \eta_{1}, \eta_{2}$ which transforms the Hamiltonian (2.1) into the following normal form:

$$
K^{I}=\frac{1}{2} \omega_{1}\left(\xi_{1}^{2}+\eta_{1}^{2}\right)+\frac{1}{2} \omega_{2}\left(\xi_{2}^{2}+\eta_{2}^{2}\right)+A\left[\xi_{2}\left(\eta_{1}^{2}-\xi_{1}^{2}\right)-2 \eta_{1} \xi_{1} \eta_{1}\right]+O_{4},
$$

where $A$ is a resonance coefficient. In the case of precise resonance $\omega_{2}=2 \omega_{1}$ this coefficient is given by the expression

$$
A=\sqrt{\frac{\left(3 \omega_{1}^{2}-1\right)^{2}\left(4 \omega_{1}^{2}-1\right)}{3 \omega_{1}^{5}}} .
$$

Following [10, 16], we introduce new time $\tau=\omega_{1} \nu$, apply a scaling canonical transformation $\xi_{i}=\varepsilon_{a x}, \eta_{i}=\varepsilon_{a y},(i=1,2)$ with valence $n=1 / \varepsilon^{2} a^{2}$ and then canonical transformations $x_{i}=\sqrt{2 r_{i}} \sin \phi_{i}, y_{i}=\sqrt{2 r_{i}} \cos \phi_{i},(i=1,2)$ and $\phi_{1}=Q_{1}, \phi_{2}=Q_{2}+2 Q_{1}, r_{1}=P_{1}-2 P_{2}, r_{2}=P_{2}$ which transform (3.13) to the form $[10,15]$

$$
K^{I I}=P_{1}+\mu P_{2}+\varepsilon\left(P_{1}-2 P_{2}\right) \sqrt{P_{2}} \sin Q_{2}+O\left(\varepsilon^{2}\right),
$$

where $\mu=\omega_{2} / \omega_{1}-2$ is a small parameter, $\varepsilon$ is a scaling coefficient and $P_{1} \geqslant 2 P_{2}$. The system with Hamiltonian (3.14) possesses a first integral $P_{1}=C$ using which and omitting the terms $O\left(\varepsilon^{2}\right)$ we arrive at a truncated system

$$
K^{I I I}=\mu P_{2}+\varepsilon\left(C-2 P_{2}\right) \sqrt{P_{2}} \sin Q_{2},
$$

which describes long-periodic motions in the neighborhood of hyperboloidal precession in the case of the third-order resonance. The system (3.15) possesses equilibria

$$
Q_{2}^{*}=\frac{\pi}{2}, \quad P_{2}^{*}=\frac{\mu+\sqrt{\mu^{2}+6 \varepsilon^{2} C}}{6 \varepsilon},
$$


and

$$
Q_{2}^{*}=-\frac{\pi}{2}, \quad P_{2}^{*}=\frac{-\mu+\sqrt{\mu^{2}+6 \varepsilon^{2} C}}{6 \varepsilon} .
$$

In the initial system with Hamiltonian (2.1) the equilibria (3.16) and (3.17) correspond to families of long-periodic motions, which we will refer to as $\Gamma_{1}$ and $\Gamma_{2}$, respectively.

Depending upon the values of the problem's parameters $\gamma, \delta$ and $h$ there may exist one or both of these families in the neighborhood of the third-order resonance curve $\gamma_{0}= \pm \frac{\sqrt{-4 \delta^{3}+17 \delta^{2}-4 \delta}}{5 \delta}$. Above the surface given by the following series of $h$

$$
\gamma(\delta, h)=\gamma_{0} \pm \frac{\sqrt{6}|3 \delta-2|(5+5 \delta)^{1 / 4}}{25 \sqrt{\delta(4-\delta)}} \sqrt{h}+O(h),
$$

which emanates from the resonant curve $\gamma_{0}$, there exist both $\Gamma_{1}$ and $\Gamma_{2}$. Below the surface (3.18) there exist one family $\Gamma_{1}$ in the case $\gamma<\gamma_{0}$ and one family $\Gamma_{2}$ in the case $\gamma>\gamma_{0}$. Due to their size we do not present the expressions for the families $\Gamma_{1}$ and $\Gamma_{2}$ in the initial variables in this work.

\subsection{Fourth-order resonance}

To obtain the long-periodic motions in the case of precise fourth-order resonance $\left(\omega_{2}=3 \omega_{1}\right)$, we apply a canonical transformation $\psi, \theta, p_{\psi}, p_{\theta} \rightarrow q_{1}, q_{2}, p_{1}, p_{2}$ that transforms the Hamiltonian (2.1) into normal form $[11,23,24]$

$$
\begin{aligned}
K^{I} & =\frac{1}{2} \omega_{1}\left(q_{1}^{2}+p_{1}^{2}\right)+\frac{1}{2} \omega_{2}\left(q_{2}^{2}+p_{2}^{2}\right)+\frac{1}{4} c_{20}\left(q_{1}^{2}+p_{1}^{2}\right)^{2}+\frac{1}{4} c_{11}\left(q_{1}^{2}+p_{1}^{2}\right)\left(q_{2}^{2}+p_{2}^{2}\right)+ \\
& +\frac{1}{4} c_{02}\left(q_{2}^{2}+p_{2}^{2}\right)^{2}-\frac{b}{4}\left[q_{1}^{2}\left(q_{1} q_{2}+3 p_{1} p_{2}\right)-p_{1}^{2}\left(p_{1} p_{2}+3 q_{1} q_{2}\right)\right]+O_{6},
\end{aligned}
$$

where $c_{11}, c_{20}, c_{02}$ are constant coefficients dependent upon the frequencies (2.5). In the case of precise fourth-order resonance they take on the following form:

$$
\begin{aligned}
& c_{11}=\frac{1}{560 \omega_{1}^{6}}\left(678 \omega_{1}^{6}+13 \omega_{1}^{4}-20 \omega_{1}^{2}+1\right), \\
& c_{20}=\frac{1}{320 \omega_{1}^{6}}\left(118 \omega_{1}^{6}-319 \omega_{1}^{4}+60 \omega_{1}^{2}-3\right), \\
& c_{02}=-\frac{1}{6720 \omega_{1}^{6}}\left(906 \omega_{1}^{6}-983 \omega_{1}^{4}-220 \omega_{1}^{2}+11\right) .
\end{aligned}
$$

In (3.19) $b$ is a resonant coefficient given by the following expression:

$$
b=\frac{1}{120} \frac{\left(1-15 \omega_{1}^{2}+56 \omega_{1}^{4}\right) \sqrt{-3+30 \omega_{1}^{2}-27 \omega_{1}^{4}}}{\omega_{1}^{6}} .
$$

We introduce new time $\tau=\omega_{1} \nu$, apply a scaling canonical transformation $q_{i}=\sqrt{\varepsilon \omega_{1} / b} x_{i}$, $p_{i}=\sqrt{\varepsilon \omega_{1} / b} y_{i},(i=1,2)$ with valence $n=b /\left(\varepsilon \omega_{1}\right)$, a canonical transformation $x_{i}=\sqrt{2 r_{i}} \sin \phi_{i}$, $y_{i}=\sqrt{2 r_{i}} \cos \phi_{i},(i=1,2)$ and omit the terms of order $\varepsilon^{2}$ and higher, which brings us to a truncated canonical system with a Hamiltonian [11, 23]

$$
K^{I I}=r_{1}+3 r_{2}+\varepsilon\left[c_{20} r_{1}^{2}+c_{11} r_{1} r_{2}+c_{02} r_{2}^{2}+r_{1}^{3 / 2} \sqrt{r_{2}} \cos \phi_{1}-3 \phi_{2}\right]
$$


and a first integral $3 r_{1}+r_{2}=$ const where $\varepsilon$ is a scaling coefficient. We apply a canonical transformation $\Psi=\phi_{1}, \Theta=\phi_{2}-3 \phi_{1}, J=3 r_{2}+r_{1}, R=r_{2}$, which transforms a system with the Hamiltonian (3.20) to the following form:

$$
\frac{d \Theta}{d \tau}=\varepsilon \frac{1}{2} \frac{\sqrt{J-3 R}(J-12 R) \cos \Theta}{\sqrt{R}}+\sigma J+\mu R, \frac{d R}{d \tau}=\varepsilon \sqrt{R}(J-12 R)^{3 / 2} \sin \Theta
$$

with a first integral $J=J_{0}=$ const where $\sigma=c_{11}-6 c_{20}$ and $\mu=c_{02}+9 c_{20}-3 c_{11}$ are constant coefficients. The system (3.21) possesses equilibria

$$
\Theta=\frac{\pi}{2} 1-\operatorname{sign}\left[\left(12 x_{*}-1\right)\left(\sigma+2 \mu x_{*}\right)\right], R=x_{*} J_{0},
$$

where $x_{*}$ is a real-value root of a cubic equation

$$
\left(432+16 \mu^{2}\right) x^{3}+(16 \mu \sigma-216) x^{2}+\left(4 \sigma^{2}+27\right) x-1=0 .
$$

For the values of the problem's parameters $\gamma$ and $\delta$ considered in this work, Eq. (3.23) possesses three real-valued roots $x_{*}$ which upon substituting into (3.22) and returning to the initial variables $\psi, \theta, p_{\psi}, p_{\theta}$ give three families of long-periodic motions that originate from the hyperboloidal precession in the case of fourth-order resonance. We refer to these families as $\Gamma_{2}, \Gamma_{3}$ and $\Gamma_{4}$. Numerical analysis carried out in [23] shows that the family $\Gamma_{2}$ obtained here coincides with the family $\Gamma_{2}$ which was obtained in the previous subsection.

\section{Bifurcation analysis of periodic motions originating from regular precessions}

Analytical representations of the families $Z_{s}, K_{s}, \Gamma_{s}, \Gamma_{i}(i=1, \ldots, 4)$ obtained in the previous section are only valid for small values of the amplitude $c$ and small deviations $\Delta h$ of the energy constant $h$ from its value on a corresponding regular precession. To obtain the existence domains of the said families for nonsmall values of $c$, a numerical method has to be used. In Refs. [14-16, 23], using a method described in [18, 22], the families $\Gamma_{s}, \Gamma_{i}(i=1, \ldots, 4)$ were continued numerically to the borders of their existence domains. Linear orbital stability of these periodic motions was studied in $[15,22,23]$. In this section we construct existence domains of the families of short-periodic motions $Z_{s}, K_{s}, \Gamma_{s}$ and consider the bifurcation problem of the families $Z_{s}, K_{s}, \Gamma_{s}$ and $\Gamma_{i}(i=1, \ldots, 4)$.

\subsection{Existence domains of periodic motions}

Using the method described in $[18,22]$ the families $Z_{s}, K_{s}, \Gamma_{s}$ of short-periodic motions arising from regular precessions of a symmetric satellite were numerically continued to the borders of their existence domains in the problem's three-dimensional parameter space. In Fig. 3 we present existence domains of the aforementioned families for fixed values of $\delta=0.5, \delta=1.0$, $\delta=2.8$ and assuming $\gamma \geqslant 0$.

The family $Z_{s}$ of short-periodic motions originating from cylindrical precession exists in a domain between the curves $S_{0}^{Z}$ and $S_{1}^{Z}$. In Fig. 3 this domain is colored gray. The family $Z_{s}$ arises from the cylindrical precession on the curve $S_{0}^{Z}$ and terminates on curve $S_{1}^{Z}$. In the case $\delta \leqslant 1.0$ the existence domain takes on the form shown in Figs. 3a, 3b. The form of the aforementioned domain in the case $\delta>1.0$ is shown in Fig. 3c. Numerical computation has 


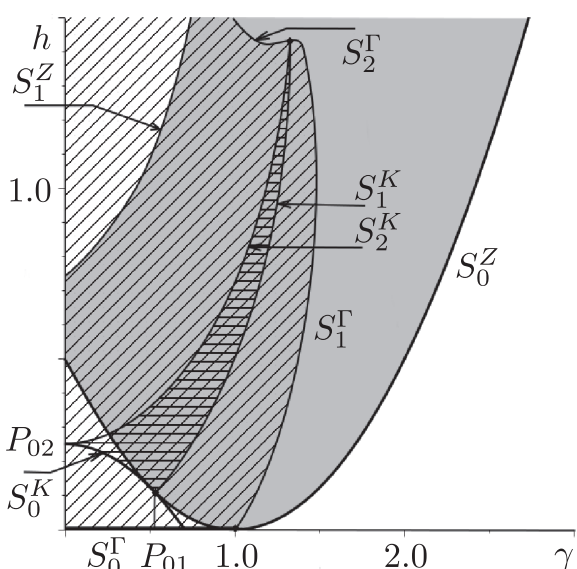

(a) Families $K_{s}, Z_{s}, \Gamma_{s}, \delta=0.5$

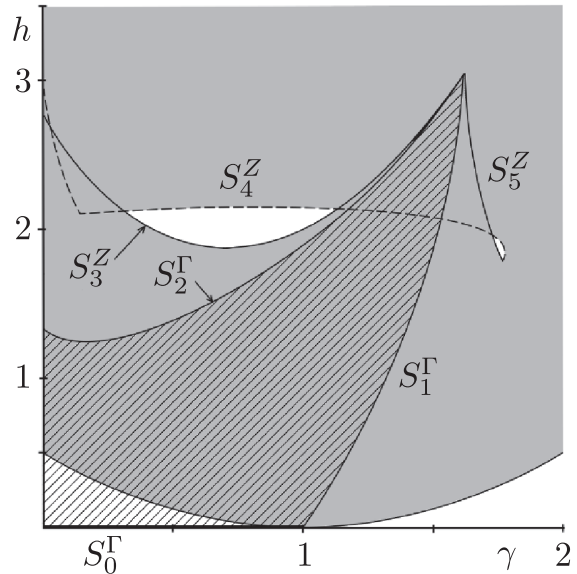

(c) Families $Z_{s}, \Gamma_{s}, \delta=2.8$

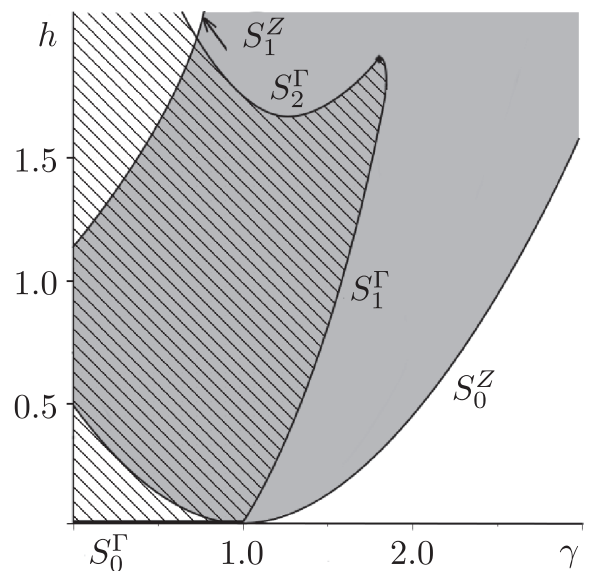

(b) Families $Z_{s}, \Gamma_{s}, \delta=1.0$

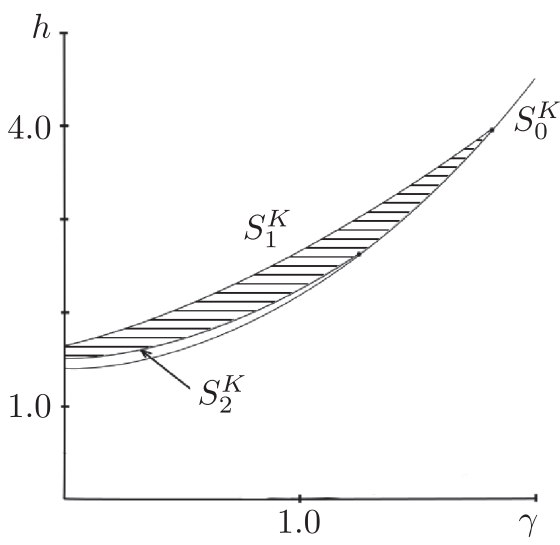

(d) Families $K_{s}, \delta=2.8$

Fig. 3. Existence domains for the families of short-periodic motions $Z_{s}$ (gray areas), $K_{s}$ (horizontally-hatched areas), $\Gamma_{s}$ (cross-hatched areas) arising from cylindrical, conical and hyperboloidal precession of a symmetric satellite.

shown that in this case the family $Z_{s}$ cannot be continued using the method described in $[18,22]$ into two subdomains enclosed by the curves $S_{3,4,5}^{Z}$.

The horizontally-hatched areas in Fig. 3 correspond to the existence domain of the family $K_{s}$ of short-periodic motions arising from conical precession. For $\delta<1.0$ (Fig. 3a) the aforementioned domain is enclosed by curves $S_{0}^{K}, S_{1}^{K}$ and $S_{2}^{K}$. Here the curve $S_{0}^{K}$ corresponds to conical precession. The family $K_{s}$ terminates on the border $S_{2}^{K}$. On the curve $S_{1}^{K}$ a bifurcation occurs and the family $K_{s}$ coincides with the family $Z_{s}$ of short-periodic motions arising from cylindrical precession. With increasing $\delta$ point $P_{01}$ of the curve $S_{1}^{K}$ approaches point $P_{02}$ and at the value $\delta=1.0$ the existence domain becomes degenerate. For $\delta>1.0$ the existence domain of the family $K_{s}$ takes on the form shown in Fig. 3d.

The cross-hatched areas in Fig. 3 correspond to the existence domain of the family $\Gamma_{s}$ of short-periodic motions arising from hyperboloidal precession. The existence domain of the family $\Gamma_{s}$ is enclosed by curves $S_{0}^{\Gamma}, S_{1}^{\Gamma}$ and $S_{2}^{\Gamma}$ where the curve $S_{0}^{\Gamma}$ corresponds to hyperboloidal precession. The family $\Gamma_{s}$ arises from hyperboloidal precession on the curve $S_{0}^{\Gamma}$ and terminates on the border $S_{2}^{\Gamma}$. The curve $S_{1}^{\Gamma}$ corresponds to the bifurcation values of the problem's param- 


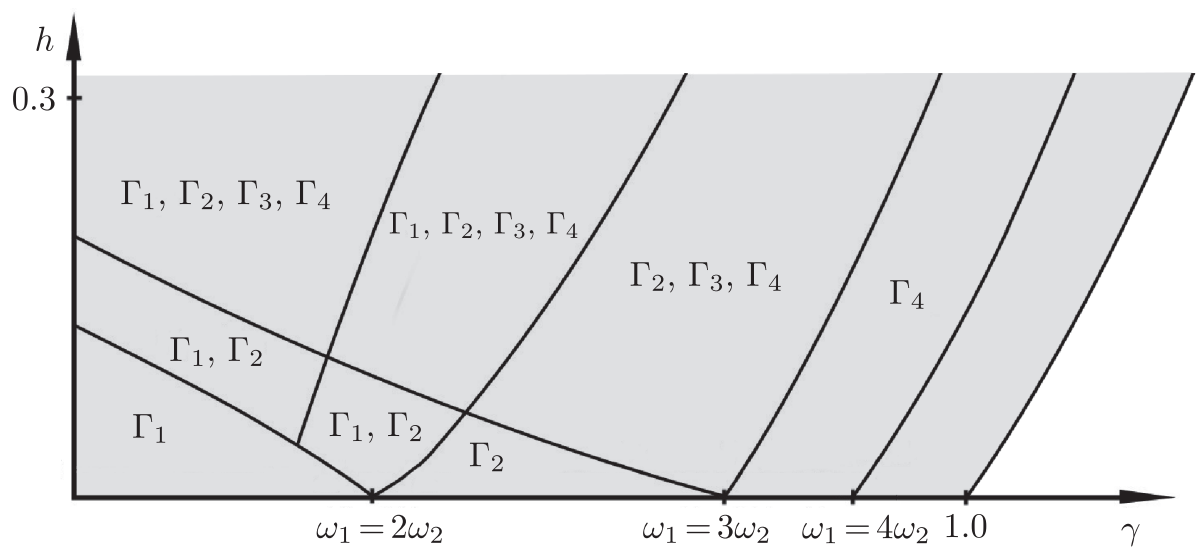

Fig. 4. Existence domains for the families of periodic motions $\Gamma_{s}$ and $\Gamma_{i}(i=1, \ldots, 4)$ emanating from hyperboloidal precession. Gray corresponds to the existence domain of the family $\Gamma_{s}$ of short-periodic motions.

eters. On $S_{1}^{\Gamma}$ the family $\Gamma_{s}$ coincides with the family $Z_{s}$ of short-periodic motions arising from cylindrical precession.

Figure 4 shows existence domains for the families of long-periodic motions $\Gamma_{i}(i=1, \ldots, 4)$ arising from hyperboloidal precession for fixed value of $\delta=1.0$. This pattern of the existence domains remains valid for $0.115<\delta<3.0$. Numerical computation shows that these domains are symmetric with respect to the axis $h$, so further results are presented assuming $\gamma>0$. In this figure the solid lines divide the parameter space into subdomains in which there may exist different numbers of long-periodic motions. On the borders of these subdomains, a bifurcation of families $\Gamma_{s}, \Gamma_{i}(i=1, \ldots, 4)$ occurs.

\subsection{Bifurcation analysis of short-periodic motions}

It has been noted above that a bifurcation of short-periodic motions originating from regular precessions may occur on the borders of their existence domains.

Figure 5 shows existence domains and a diagram of periods $T_{K}, T_{Z}, T_{\Gamma}$ of the families $Z_{s}$, $K_{s}, \Gamma_{s}$ for fixed values of $\delta=0.5$ and $h=0.35$. In Fig. 5b the solid lines represent intervals of linear orbital stability and the dashed lines represent intervals of orbital instability of the aforementioned families of periodic motions. The method of investigating the linear orbital stability of the said periodic motions is described in Refs. [19, 22].

For $\gamma<0.16$ there exist only one family of linear orbitally stable short-periodic motions $-\Gamma_{s}$. At the value $\gamma=0.16$ a family of linear orbitally stable short-periodic motions $Z_{s}$ arises from cylindrical precession and at the value $\gamma=0.42$ a family of orbitally unstable short-periodic motions $K_{s}$ arises from conical precession. With increasing $\gamma$ the periods $T_{K}$ and $T_{Z}$ of the families $K_{s}$ and $Z_{s}$ converge and coincide at the point $B_{1}(\gamma=0.78)$. At this point a bifurcation occurs: the family $K_{s}$ coincides with $Z_{s}$ and periodic motions of the family $Z_{s}$ become orbitally unstable. With further growth of parameter $\gamma$ the period $T_{\Gamma}$ of the family $\Gamma_{s}$ converges with the period $T_{Z}$ of the family $Z_{s}$ and these two families coincide at the point $B_{2}(\Gamma=1.11)$. To the right of the point $B_{2}$ only one family of short-periodic motions remains - the family $Z_{s}$ of short-periodic motions arising from cylindrical precession. Periodic motions of the family $Z_{s}$ are linear orbitally stable for $\gamma>1.11$. The family $Z_{s}$ coincides with cylindrical precession at point $P_{3}(\gamma=1.64)$. This bifurcation pattern remains valid for $0<\delta<1$.0. 


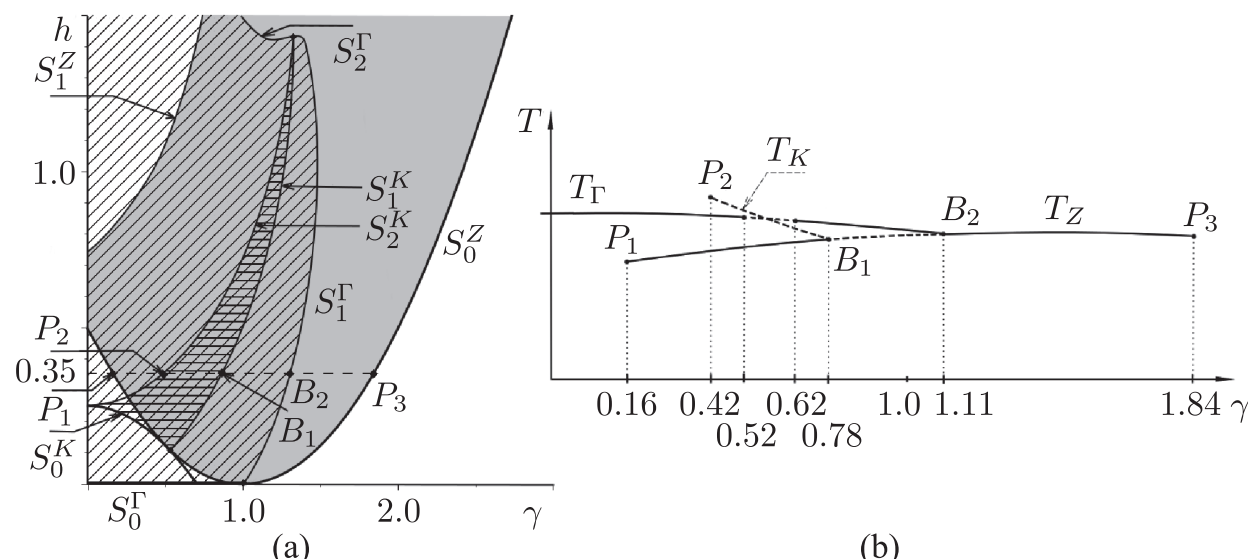

(a)

(b)

Fig. 5. Existence domains and a bifurcation diagram of the families $Z_{s}, K_{s}, \Gamma_{s}$ of short-periodic motions emanating from cylindrical, conical and hyperboloidal precession for fixed values of $\delta=0.5, h=0.35$. Here $T_{Z}, T_{K}, T_{\Gamma}$ are periods of the periodic motions belonging to the families $Z_{s}, K_{s}, \Gamma_{s}$, respectively.

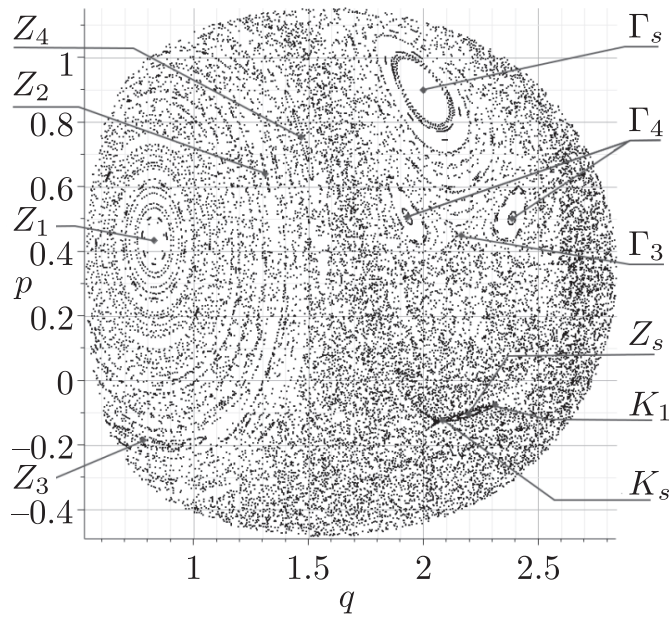

(a)

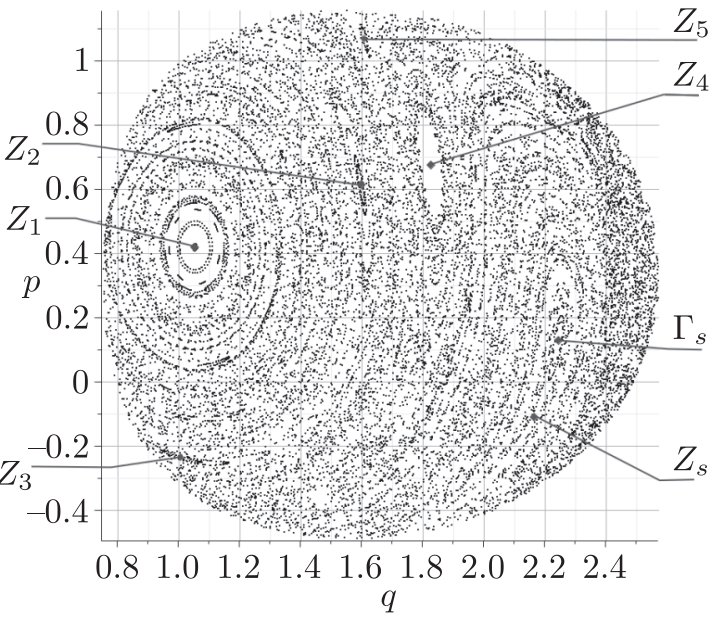

(b)

Fig. 6. Poincaré maps computed in the neighborhood of bifurcation points $B_{1}$ (a) and $B_{2}$ (b) for fixed values of $\gamma=0.77$ and $\gamma=1.09$, respectively.

To verify the results described above, Poincaré maps were constructed in the neighborhood of the bifurcation points $B_{1}$ and $B_{2}$. Figure 6 a shows a map constructed near the point $B_{1}$ for the values $\gamma=0.77, \delta=0.5, h=0.35$. On this Poincaré map a point marked $Z_{s}$ corresponds to a linear orbitally stable short-periodic motion of the family $Z_{s}$ and the point marked $K_{s}$ corresponds to an orbitally unstable short-periodic motion of the family $K_{s}$. With $\gamma$ approaching the bifurcation value the points corresponding to the families $K_{s}$ and $Z_{s}$ converge and coincide forming a point corresponding to an orbitally unstable periodic motion of the family $Z_{s}$ (Fig. 6b).

Figure $6 \mathrm{~b}$ shows a Poincaré map constructed near the point $B_{2}$ for the values $\gamma=1.09$, $\delta=0.5, h=0.35$. On this map the point marked $Z_{s}$ corresponds to an orbitally unstable short-periodic motion of the family $Z_{s}$ and the point marked $\Gamma_{s}$ corresponds to a linear orbitally stable short-periodic motion of the family $\Gamma_{s}$. With $\gamma$ approaching the bifurcation value at point $B_{2}$ the points corresponding to the families $Z_{s}$ and $\Gamma_{s}$ converge and coincide forming a point corresponding to a linear orbitally stable periodic motion of the family $Z_{s}$ (Fig. 7). 


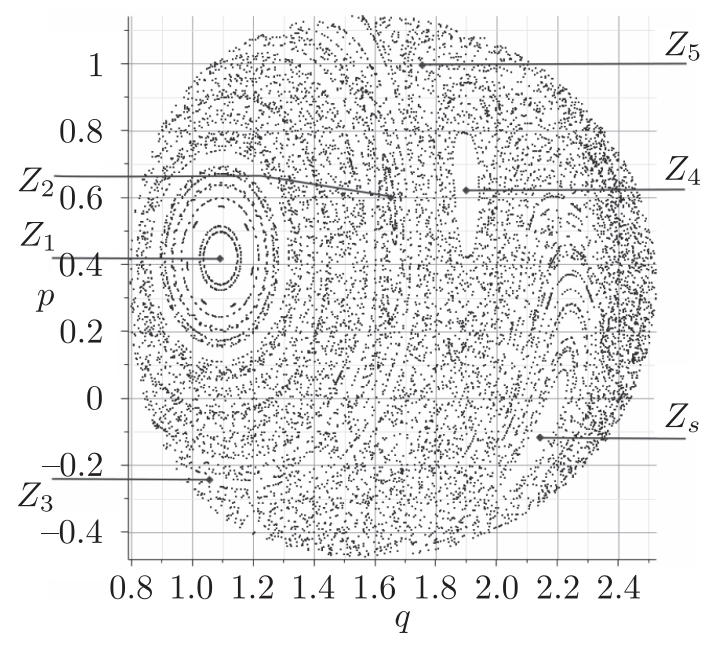

Fig. 7. Poincaré map computed in the neighborhood of the bifurcation point $B_{2}$ for fixed value of $\gamma=1.15$.

In Fig. 6 and Fig. 7 there also exist points marked $Z_{i}(i=1, \ldots, 5), K_{1}, \Gamma_{3,4}$ corresponding to long-periodic motions arising from regular precessions. The families $\Gamma_{3,4}$ of long-periodic motions arising from hyperboloidal precession have been obtained analytically and numerically in [22]. Numerical computation shows that the families $Z_{i}(i=1, \ldots, 5)$ and $K_{1}$ arise from cylindrical and conical precessions, respectively.

\subsection{Bifurcation analysis of long-periodic motions originating from hyperboloidal precession}

Figures 8 and 10 show bifurcation diagrams of periodic motions originating from the hyperboloidal precession of a satellite for $\delta=1.0$ and different values of the energy constant $h$. In these diagrams the periods $T_{s}, T_{i}(i=1, \ldots, 4)$ of the families $\Gamma_{s}, \Gamma_{i}(i=1, \ldots, 4)$ are mapped against the parameter $\gamma$ for fixed values of $h$. The curves $N T_{s}$ represent period $T_{s}$ of the family $\Gamma_{s}$ multiplied $N$ times. The solid lines represent intervals of linear orbital stability, while gray lines represent intervals of orbital instability.

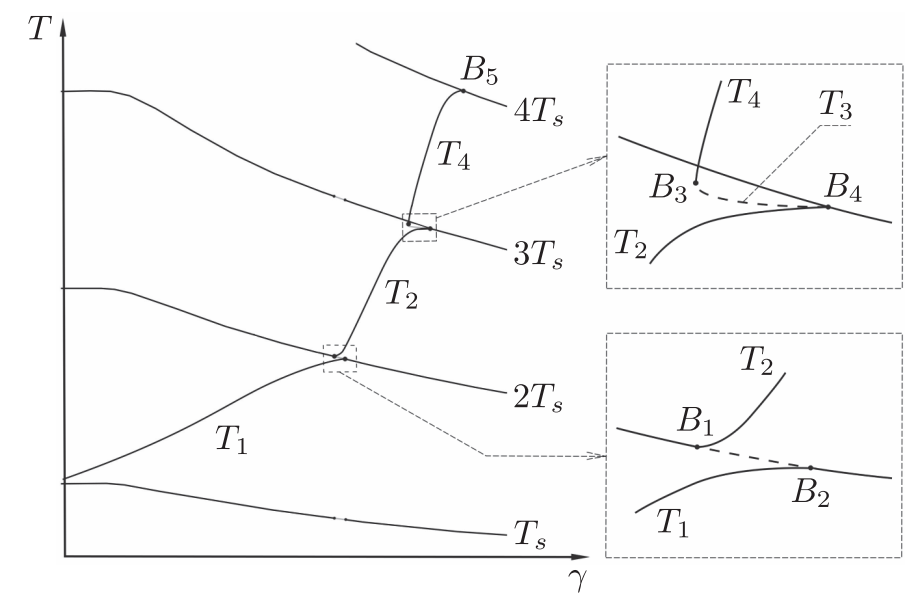

Fig. 8. Bifurcation diagrams for families of periodic motions originating from the hyperboloidal precession of a symmetric satellite obtained for fixed values of $h=0.001, \delta=1.0$ and $h=0.1, \delta=1.0$. 
Figures 8 show a diagram for $h=0.001$ which represents relatively small deviations of the energy constant from its value on hyperboloidal precession and corresponds to the results presented in the analytical studies [10,13]. In this case, for small values of $\gamma$ there exist a family of short-periodic motions $\Gamma_{s}$ and a family of long-periodic motions $\Gamma_{1}$. At point $B_{1}$ a family of long-periodic motions $\Gamma_{2}$ branches off from the family $\Gamma_{s}$, so between the points $B_{1}$ and $B_{2}$ there exist two families of long-periodic motions which arise from hyperboloidal precession. At point $B_{2}$ the period $T_{1}$ of the family $\Gamma_{1}$ becomes twice as large as the period $T_{s}$ of the family $\Gamma_{s}$ and the families $\Gamma_{1}$ and $\Gamma_{s}$ coincide. The family $\Gamma_{2}$ can be numerically continued to the point $B_{4}$ where it coincides with $\Gamma_{s}$. At this point the period $T_{2}$ becomes equal to $3 T_{s}$. At point $B_{3}$ two families arise: $\Gamma_{3}$ and $\Gamma_{4}$. The family $\Gamma_{3}$ can be continued to the point $B_{4}$ where it coincides with $\Gamma_{s}$. Upon approaching point $B_{3}$ the period $T_{3}$ gets closer to $3 T_{S}$. The family $\Gamma_{4}$ coincides with $\Gamma_{s}$ at point $B_{5}$ with its period $T_{4}$ becoming equal to $4 T_{s}$. The family $\Gamma_{s}$ can be continued along the $\gamma$ axis to the value $\gamma \approx 1.01$ where it coincides with a family of periodic motions that arises from cylindrical precession.

Figure 9 shows Poincaré maps computed for fixed values of $h=0.001$ and $\delta=1.0$ in the neighborhood of the bifurcation point $B_{1}$. The left map shows families $\Gamma_{s}$ and $\Gamma_{1}$ before the bifurcation. The right map shows the family $\Gamma_{2}$ branching off from $\Gamma_{s}$ with $\Gamma_{s}$ becoming orbitally unstable.
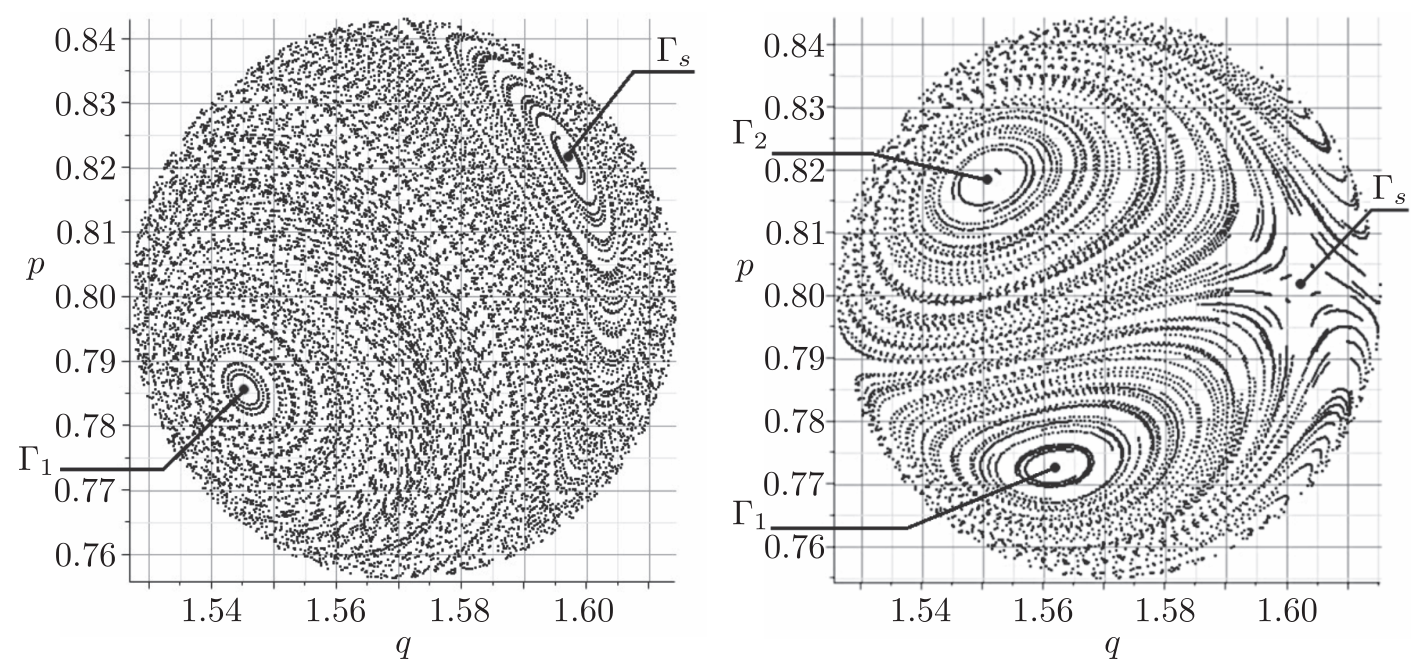

Fig. 9. Poincaré maps computed for fixed values of $h=0.001$ and $\delta=1.0$ in the neighborhood of the bifurcation point $B_{1}$ shown in Fig. 3a.

Figure 10a shows a diagram for $h=1.0$. In this case, for small values of $\gamma$ there exist a family $\Gamma_{s}$ and a family $\Gamma_{s}$. At point $B_{1}^{*}$ families $\Gamma_{1}^{*}$ and $\Gamma_{2}$ appear. The family $\Gamma_{1}^{*}$ exists for $h>0.05$ and has been obtained numerically using Poincaré maps. With increasing $\gamma$ the period $T_{1}$ becomes closer to $2 T_{s}$ and at point $B_{2}$ the families $\Gamma_{1}$ and $\Gamma_{s}$ coincide. $\Gamma_{2}$ coincides with $\Gamma_{s}$ at point $B_{3}$ with its period $T_{2}$ becoming equal to $2 T_{s}$. Approaching the point $B_{1}$, period $T_{1}^{*}$ of the family $\Gamma_{1}^{*}$ gets closer to $2 T_{s}$ and at point $B_{1}$ the family $\Gamma_{1}^{*}$ coincides with $\Gamma_{s}$.

At point $B_{3}$ two families of long-periodic motions arise: $\Gamma_{3}$ and $\Gamma_{4}$. The family $\Gamma_{3}$ can be continued to the point $B_{4}$ where it coincides with $\Gamma_{s}$ and its period $T_{3}$ becomes equal to $3 T_{s}$. The family $\Gamma_{4}$ coincides with $\Gamma_{s}$ at point $B_{5}$ where its period $T_{4}$ equals $T_{s}$. The family $\Gamma_{s}$ can be continued along the $\gamma$ axis to the value $\gamma \approx 1.04$ where it coincides with a family of periodic motions that arises from cylindrical precession. 


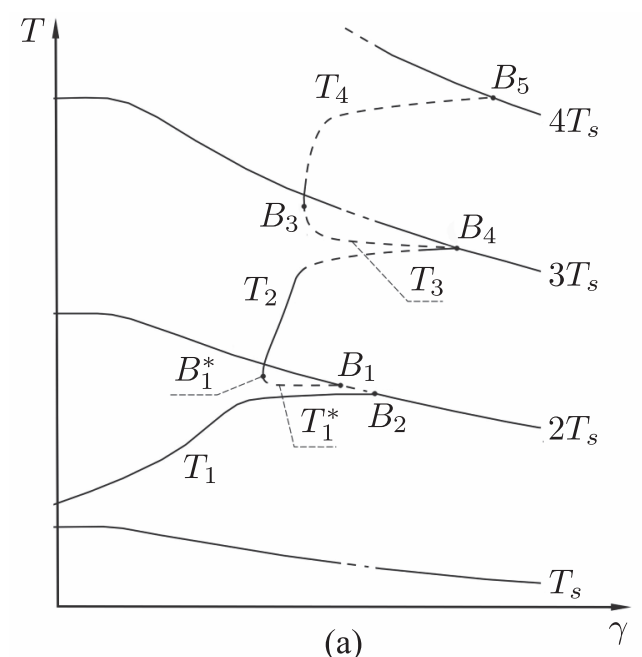

(a)

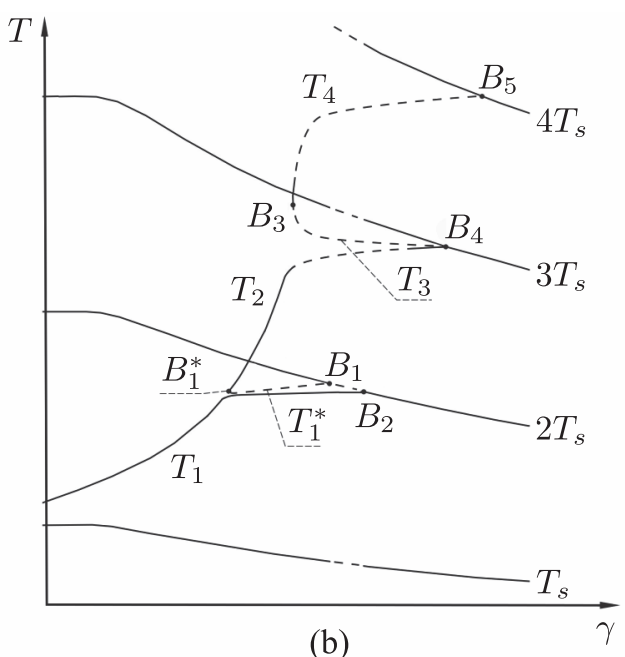

(b)

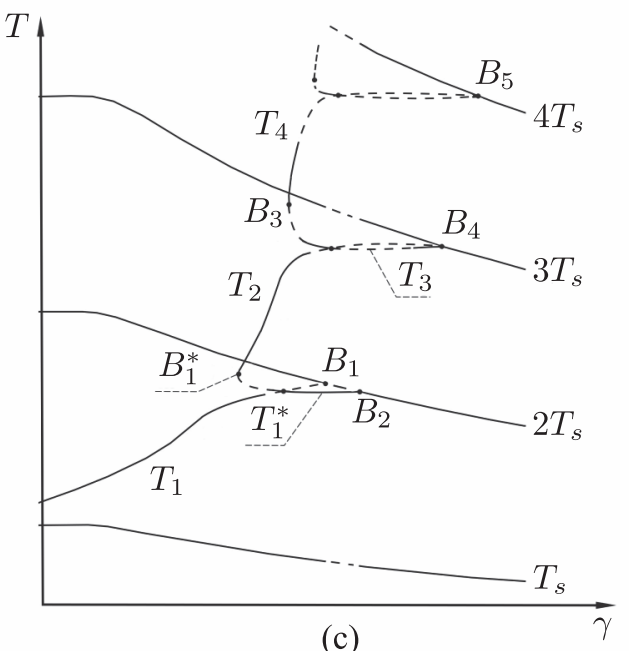

(c)

Fig. 10. Bifurcation diagrams for families of periodic motions originating from the hyperboloidal precession of a symmetric satellite obtained for fixed values of $h=0.205, \delta=1.0$ and $h=0.3, \delta=1.0$.

The diagram shown in Fig. 10b represents the case of $h=0.205$. In the interval $0.204<h<0.206$ there exists a value $h^{*}$ for which the curves $T_{1}^{*}$ and $T_{2}$ form a sharp angle at the point $B_{1}^{*}$ which coincides with the curve $T_{1}$. For $h>h^{*}$ the family $\Gamma_{1}^{*}$ takes the place of $\Gamma_{1}$ and coincides with $\Gamma_{s}$ at the point $B_{2}$, while the family $\Gamma_{1}$ coincides with $\Gamma_{s}$ at the point $B_{1}$. The families $\Gamma_{2}, \Gamma_{3}$ and $\Gamma_{4}$ retain similar behavior as in the previous case. A similar change in the bifurcation diagram occurs at the value $h \approx 0.25$ where the curves $T_{2}, T_{3}$ and $T_{4}$ converge at the point $B_{3}$ and the families $\Gamma_{2}$ and $\Gamma_{3}$ switch places. Figure 10c shows a diagram computed for $h=0.3$ which demonstrates the aforementioned change.

\section{Conclusion}

In this work we have constructed families of periodic motions emanating from the regular precession of a dynamically symmetric satellite for all admissible values of the problem parameters. A bifurcation problem for the said families of periodic motions has been addressed. The results have been presented in the form of bifurcation diagrams and Poincaré maps. 


\section{References}

[1] Duboshin, G. N., On Rotational Movement Of Artificial Celestial Bodies, Bull. ITA AN SSSR, 1960, vol. 7, no. 7, pp. 511-520 (Russian).

[2] Kondurar, V. T., Particular Solutions of the General Problem of the Translational-Rotational Motion of a Spheroid Attracted by a Sphere, Sov. Astron., 1960, vol.3, no. 5, pp. 863-875; see also: Astron. Zh., 1959, vol.36, no. 5, pp. 890-901.

[3] Beletsky, V. V., Motion of a Satellite about Its Center of Mass in the Gravitational Field, Moscow: MGU, 1975 (Russian).

[4] Chernous'ko, F. L., On the Stability of Regular Precession of a Satellite, J. Appl. Math. Mech., 1964, vol. 28, no. 1, pp. 181-184; see also: Prikl. Mat. Mekh., 1964, vol. 28, no. 1, pp. 155-157.

[5] Lyapunov, A. M., The General Problem of the Stability of Motion, London: Fracis \& Taylor, 1992.

[6] Sokol'skiy, A. G. and Khovanskiy, S. A., Periodic Motions Close to Hyperboloidal Precession of a Symmetric Satellite in Circular Orbit, Kosmicheskie Issledovaniya, 1979, vol. 17, no. 2, pp. 208217 (Russian).

[7] Markeev, A. P., Linear Hamiltonian Systems and Some Problems of Stability of the Satellite Center of Mass, Izhevsk: R\&C Dynamics, Institute of Computer Science, 2009 (Russian).

[8] Markeev, A. P. and Bardin, B. S., On the Stability of Planar Oscillations and Rotations of a Satellite in a Circular Orbit, Celest. Mech. Dynam. Astronom., 2003, vol. 85, no. 1, pp. 51-66.

[9] Bardin, B.S., On Orbital Stability of Planar Motions of Symmetric Satellites in Cases of First and Second Order Resonances, in Proc. of the 6th Conference on Celestial Mechanics (Señorio de Bértiz, 2003), J. Palacian, P. Yanguas (Eds.), Monogr. Real Acad. Ci. Exact. Fís.-Quím. Nat. Zaragoza, vol. 25, Zaragoza: Real Acad. Ci. Exact. Fís.-Quím. Nat., 2004, pp. 59-70.

[10] Markeyev, A.P., Non-Linear Oscillations of a Hamiltonian System with 2 : 1 Resonance, J. Appl. Math. Mech., 1999, vol.63, no.5, pp.715-726; see also: Prikl. Mat. Mekh., 1999, vol.63, no.5, pp. $757-769$.

[11] Bardin, B. S. and Chekin, A. M., Non-Linear Oscillations of a Hamiltonian System in the Case of $3: 1$ Resonance, J. Appl. Math. Mech., 2009, vol. 73, no.3, pp. 249-258; see also: Prikl. Mat. Mekh., 2009, vol. 73, no. 3, pp. 353-367.

[12] Bardin, B.S., On Nonlinear Motions of Hamiltonian System in Case of Fourth Order Resonance, Regul. Chaotic Dyn., 2007, vol.12, no.1, pp.86-100.

[13] Bardin, B.S., On the Orbital Stability of Periodic Motions of a Hamiltonian System with Two Degrees of Freedom in the case of $3: 1$ Resonance, J. Appl. Math. Mech., 2007, vol.71, no.6, pp. 880-891; see also: Prikl. Mat. Mekh., 2007, vol.71, no.6, pp.976-988.

[14] Sukhov, E. A. and Bardin, B. S., Numerical Analysis of Periodic Motions of a Dynamically Symmetric Satellite Originated from Its Hyperboloidal Precession, Inzhenern. Zhurnal: Nauka i Innovatsii, 2016, no. 5(53), 10 pp. (Russian).

[15] Sukhov, E. A. and Bardin, B. S., On Periodic Motions Originating from Hyperboloidal Precession on a Symmetric Satellite, in Abstracts of the 13th All-Russian Conf. on Problems of Dynamics, Physics of Plasma Particles and Optoelectronics (Moscow, RUDN, 2017).

[16] Sukhov, E. A. and Bardin, B.S., Numerical and Analytical Plotting of Periodic Motion and Investigating Motion Stability in the Case of a Symmetric Satellite, Inzhenern. Zhurnal: Nauka i Innovatsii, 2017, no. 11(71), 3 pp. (Russian).

[17] Deprit, A. and Henrard, J., Natural Families of Periodic Orbits, Astron. J., 1967, vol.72, no. 2, pp. $158-172$.

[18] Karimov, S. R. and Sokolskiy, A. G., Method of Numerical Continuation of Natural Families of Periodic Motions of Hamiltonian Systems, Preprint No. 9, Moscow: Institute of Theoretical Astronomy of the Academy of Sciences of the USSR, 1990. 
[19] Sokolskiy, A. G. and Khovanskiy, S. A., On Numerical Continuation of Periodic Motions of a Lagrangian System with Two Degrees of Freedom, Kosmicheskie Issledovaniya, 1983, vol.21, no.6, pp. 851-860 (Russian).

[20] Lara, M., Deprit, A., and Elipe, A., Numerical Continuation of Families of Frozen Orbits in the Zonal Problem of Artificial Satellite Theory, Celestial Mech. Dynam. Astronom., 1995, vol.62, no. 2, pp. 167-181.

[21] Lara, M. and Peláez, J., On the Numerical Continuation of Periodic Orbits: An Intrinsic, 3-Dimentional, Differential, Predictor-Corrector Algorithm, Astron. Astrophys., 2002, vol. 389, no. 2, pp. 692-701.

[22] Sukhov, E. A. and Bardin, B. S., On the Algorithm for Numerical Computation of Periodic Motions of a Hamiltonian System with Two Degrees of Freedom, in Abstracts of the 14th All-Russian Conf. on Problems of Dynamics, Physics of Plasma Particles and Optoelectronics (Moscow, RUDN, 2018).

[23] Sukhov, E. A., Analytical and Numerical Computation and Study of Long-Periodic Motions Originating from Hyperboloidal Precession of a Symmetric Satellite, in Proc. of hte 8th Polyahov Readings (St. Petersburg, St. Petersburg State University, 2018).

[24] Schmidt, D.S., Periodic Solutions near a Resonant Equilibrium of a Hamiltonian System, Celestial Mech., 1974, vol. 9, pp. 81-103. 